\title{
REAL ANALYTIC MAXIMUM MODULUS MANIFOLDS IN STRICTLY PSEUDOCONVEX BOUNDARIES
}

\author{
ANDREI IORDAN \\ Université Pierre et Marie Curie Paris VI, Mathématiques \\ 4, Place Jussieu, 75252 Paris Cedex 05, France
}

1. Introduction. Let $D$ be a domain in $\mathbb{C}^{n}$. We shall denote by $A^{k}(D)$ the algebra of the holomorphic functions in $D$ which have a $C^{k}$ extension to $\bar{D}$ and by $\mathcal{O}(\bar{D})$ the algebra of the holomorphic functions in a neighborhood of $\bar{D}$. A subset $E$ of $\partial D$ is locally a maximum modulus set for $A^{k}(D)\left(\left(L M A^{k}\right)\right.$ for short $)$ if for every $p \in \partial D$, there exist a neighborhood $U$ of $p$ and $f \in A^{k}(D \cap U)$ such that $|f|=1$ on $E \cap U$ and $|f|<1$ on $\bar{D} \cap U \backslash E$. Similarly, $E$ is locally a peak set for $A^{k}(D)\left(\left(L P A^{k}\right)\right.$ for short) if for every $p \in E$, there exist a neighborhood $U$ of $p$ and $f \in A^{k}(D \cap U)$ such that $f=1$ on $E \cap U$ and $|f|<1$ on $\bar{D} \cap U \backslash E$. We have the same definitions for the algebra $\mathcal{O}(\bar{D})$ of the holomorphic functions in a neighborhood of $\bar{D}((L M H)$ and $(L P H)$ for short).

The characterization of the subsets of the boundary of a bounded strictly pseudoconvex domain with $C^{\infty}$ boundary which are $\left(L P A^{\infty}\right)$ is well known: these are sets which are locally contained in totally real complex-tangential submanifolds of dimension $n-1$ ([HS] and [CC2]). In fact, these sets are also global peak sets for $A^{\infty}(D)[\mathrm{FH}]$.

For instance, few things are known about the sets which are $\left(L M A^{k}\right)$ and are not $\left(L P A^{k}\right)$. The situation is clear only for the real analytic submanifolds $M$ of dimension $n$ in the boundary of strictly pseudoconvex domains with real analytic boundary: $M$ is $(L M H)$ if and only if $M$ is totally real and admits a real analytic foliation by complex-tangential submanifolds of codimension 1 [DS]. In general, a subset $E$ of the boundary of a strictly pseudoconvex domain with $C^{\infty}$ boundary which is $\left(L M A^{\infty}\right)$ is locally contained in totally real submanifolds of dimension $n$ which admit a foliation of dimension 1 which is complex-tangential at the points of $E$ [I2]. But a set which is $(L M H)$ is not in general a global maximum modulus set

1991 Mathematics Subject Classification: 32E25, 32F15, 32F25.

The paper is in final form and no version of it will be published elsewhere. 
[DS] and a submanifold of dimension $n$ of the boundary of a strictly pseudoconvex domain with real analytic boundary which is $\left(L M A^{2}\right)$ is real analytic [NR].

Here we use the contact structure of the boundary of a strictly pseudoconvex domain to study the foliations by complex-tangential submanifolds [BI]. These methods were used in the context of interpolation sets, peak sets or maximum modulus sets in $[\mathrm{HT}],[\mathrm{CC} 1],[\mathrm{CC} 2]$ and [DS]. We present the results from [BS] and $[\mathrm{DS}]$ about real analytic submanifolds of maximal dimension of the boundary of a strictly pseudoconvex domain with real analytic boundary which are $(L M H)$ and we use them to obtain results in lower dimension ([BI]). Finally we present an approach from [NR] (matching of holomorphic and antiholomorphic functions along maximum modulus sets) and some examples from [NR], which prove that the situation is really complicated for curves transverse to the complex-tangent space which are not real analytic.

\section{Preliminaries}

a) Symplectic structures. A symplectic manifold is a couple $(X, \Omega)$, where $X$ is a differentiable manifold of dimension $2 n$ and $\Omega$ is a 2 closed form on $X$ such that $\Omega^{n} \neq 0$ on $X$. A submanifold $M$ of $X$ is isotropic if $\Omega(\xi, \eta)=0$ for all $\xi, \eta$ tangent to $M$. If $M$ is an isotropic submanifold of $X$, we have $\operatorname{dim} M \leq n$ and if $\operatorname{dim} M=n$ we say that $M$ is lagrangian.

We shall use the following:

Theorem 1 (Darboux-Weinstein theorem) [WEI]. Let $\left(X_{1}, \Omega_{1}\right),\left(X_{2}, \Omega_{2}\right)$, two symplectic manifolds of the same dimension, $M_{1} \subset X_{1}, M_{2} \subset X_{2}$, submanifolds. Let $\varphi: M_{1} \rightarrow M_{2}$ a diffeomorphism such that $\varphi^{*}\left(\Omega_{2} \mid M_{2}\right)=\Omega_{1} \mid M_{1}$. Then, for every $p \in M_{1}$ there exist a neighborhood $V_{1}$ in $X_{1}$, a diffeomorphism $\psi$ on a neighborhood $V_{2}$ of $\varphi(p)$ in $X_{2}$ such that $\psi$ is an extension of $\varphi$ and $\psi^{*}\left(\Omega_{2} \mid V_{2}\right)=$ $\Omega_{1} \mid V_{1}$.

Theorem 1 is also true for real analytic objects.

b) Contact structures. A contact manifold is a couple $(Z, \omega)$, where $Z$ is a differentiable manifold of dimension $2 n+1$, and $\omega$ is a 1 form on $Z$ such that $\omega \wedge(d \omega)^{n} \neq 0$ on $Z$. There exists a unique vector field $X_{\omega}$ on $Z$ (the characteristic vector field) such that $i\left(X_{\omega}\right) \omega=1, i\left(X_{\omega}\right) d \omega=0$, where $i(\xi) \eta$ is the left inner product of a differential form $\eta$ by a vector field $\xi$.

A submanifold $N$ of $Z$ is isotropic if $\omega \mid N=0$. If $N$ is an isotropic submanifold of $Z$, we have $\operatorname{dim} N \leq n$ and if $\operatorname{dim} N=n$, we say that $N$ is a Legendre manifold.

c) Levi form. Let $D$ be a strictly pseudoconvex domain with $C^{2}$ boundary and $\rho$ a strictly plurisubharmonic defining function for $D$. We denote by $j$ the inclusion of $\partial D$ in $\mathbb{C}^{n}$ and $\omega=j^{*}\left(\frac{1}{i} \partial \rho\right)$. Then the complex-tangent space to $\partial D$ is $T^{c}(\partial D)=\operatorname{ker} \omega$ and $\omega$ is a contact form on $\partial D$.

If $\xi, \eta$ are sections of $T^{c}(\partial D)$, the Levi form is defined by

$$
\mathcal{L}(\xi, \eta)=\partial \bar{\partial} \rho(X, \bar{Y})
$$


where $X, Y \in T^{c}(\partial D) \otimes \mathbb{C}, \xi=\operatorname{Re} X, \eta=\operatorname{Re} Y$. The form $\mathcal{L}(\xi, \eta)$ defines a hermitian metric on $T^{c}(\partial D)$ and we shall say that $\xi$ and $\eta$ are $\mathcal{L}$-orthogonal if $\mathcal{L}(\xi, \eta)=0$.

A submanifold $M$ of $\partial D$ is complex-tangential if $M$ is an isotropic submanifold of the contact manifold $(\partial D, \omega)$. Since $\partial \bar{\partial} \rho \mid \partial D=-i d \omega$ a complex-tangential submanifold $M$ is totally real and $\operatorname{dim} M \leq n-1$ [HT], [BS]. Also we have $d \omega(\xi, \eta)=-\frac{1}{2} \operatorname{Im} \mathcal{L}(\xi, \eta)$.

\section{Isotropic foliations}

Proposition $1[\mathrm{BI}]$. Let $(X, \omega)$ be a contact manifold of dimension $2 n+1$ and $M$ an isotropic submanifold of dimension $k, 0 \leq k \leq n$. Then, for every $p \in M$ there exist local coordinates $\left(x_{0}, \ldots, x_{2 n}\right)$ in a neighborhood of $p$ such that $\omega=d x_{0}+\sum_{1}^{n} x_{i} d x_{i+n}$ and $M=\left\{x_{0}=x_{k+1}=\ldots=x_{2 n}=0\right\}$. In particular $M$ is an intersection of Legendre submanifolds.

Proof. Let $p \in M$. Since the characteristic vector field $X_{\omega}$ is transverse to $M$, we may find a neighborhood $U$ of $p$ such that $Y=U / X_{\omega}$ is a manifold and the restriction of the projection $\pi: U \rightarrow Y$ to $M$ is an diffeomorphism onto $\pi(M \cap U)$. Then $(Y, \sigma)$ is a symplectic manifold, where $\sigma$ is the form induced by $d \omega$ on $Y$. Since $\pi(M \cap U)$ is isotropic, by theorem 1 we may extend a coordinate system of $\pi(M \cap U)$ to a coordinate system $\left(\widetilde{x}_{1}, \ldots, \widetilde{x}_{2 n}\right)$ on $Y$ such that $\pi(M \cap U)=\left\{\widetilde{x}_{k+1}=\ldots=\widetilde{x}_{2 n}=0\right\}$ and $\sigma=\sum_{1}^{n} d \widetilde{x}_{i} \wedge d \widetilde{x}_{i+n}$. Then, if $\widetilde{x}_{i}=x_{i} \circ \pi$, the form $\omega-\sum_{1}^{n} x_{i} \wedge d x_{i+n}$ is closed and we may find $x_{0}$ such that $\omega=d x_{0}+\sum_{1}^{n} x_{i} d x_{i+n}$ and $M=\left\{x_{0}=x_{k+1}=\ldots=x_{2 n}=0\right\}$.

Now, $M$ is the intersection of the Legendre manifolds $\left\{x_{0}=x_{k+1}=\ldots=\right.$ $\left.x_{k+n}=0\right\}$ and $\left\{x_{0}=x_{n+1}=\ldots=x_{2 n}=0\right\}$.

Proposition $2[\mathrm{BI}]$. Let $(X, \omega)$ be a contact manifold of dimension $2 n+1$ and $M$ a submanifold of dimension $k+1$ transverse to $\operatorname{ker} \omega$. Then $M$ admits a foliation by isotropic submanifolds of codimension 1 if and only if there exist local coordinates $\left(x_{0}, \ldots, x_{2 n}\right)$ such that $\omega=\varphi\left(d x_{0}+\sum_{1}^{n} x_{i} d x_{i+n}\right)$, with $\varphi \neq 0$ and $M=\left\{x_{k+1}=\ldots=x_{2 n}=0\right\}$. In particular, in this case, $M$ is intersection of $n+1$ dimensional submanifolds foliated by Legendre submanifolds.

Proof. If $\omega$ is as in proposition 2, it is clear that the submanifolds $M_{c}=$ $\left\{x_{k+1}=\ldots=x_{2 n}=0, x_{0}=c\right\}$ give an isotropic foliation of codimension 1 of $M$, so we have only to prove the converse.

Let $p \in M$. By Frobenius theorem, there exist a neighborhood $U$ of $p$ and functions $f, u$ on $U$ such that $j^{*}(f \omega-d u)=0$ on $U$, where $j: M \rightarrow X$ is the inclusion. Since $M$ is transverse to $\operatorname{ker} \omega$, there exists a vector field $\xi$ tangent to $M$ in a neighborhood of $p$ such that $i(\xi) j^{*}(f \omega)=1$.

We shall consider $\omega^{\prime}=\varphi \omega$ such that the restriction of $X_{\omega^{\prime}}$ to $M$ is $\xi$.

For this we shall prove that there exists a function $g$ in the neighborhood of $p$ such that $g=1$ on $M$ and $i(\xi) d(g f \omega)=0$ on $M$. Indeed, since $j^{*} d(f \omega)=0$ and $\xi$ 
is tangent to $M$, we have $i(\xi) j^{*} d(f \omega)=0$. It follows that $i(\xi) d(f \omega)$ is of the form

$$
\sum \alpha_{r} d u_{r}+u_{r} \beta_{r}
$$

where $\alpha_{r}, \beta_{r}$ are 0 and respectively 1 forms defined in a neighborhood of $p$ and $u_{r}=0$ on $M$. We may take $g=1+\sum \alpha_{r} u_{r}$.

Then, by taking $\varphi=f g$, since the characteristic vector field is the unique vector field $\eta$ which satisfies $i(\eta) \omega^{\prime}=1$ and $i(\eta) d \omega^{\prime}=0$, we have $X_{\omega^{\prime}}=\xi$ on a neighborhood of $p$ in $M$. Since $i(\eta) \omega^{\prime}=1$ and $\omega^{\prime}-d u|M=f \omega-d u| M=0$ there exists an extension $\widetilde{u}$ of $u$ such that $i\left(X_{\omega^{\prime}}\right) d \widetilde{u}=1$ and we consider the foliation of $X$ given by $\widetilde{u}=$ constant.

Since $i\left(X_{\omega^{\prime}}\right) d \widetilde{u}=1$, there exists a diffeomorphism $x \rightarrow(t, y)$ from a neighborhood $V$ of $p$ to $I \times Y$, where $I$ is a real interval and $Y$ is the manifold of orbits, such that $X_{\omega^{\prime}}$ is transformed to $\frac{\partial}{\partial t}$. Since $d \omega^{\prime}$ is an absolute integral invariant of $X_{\omega^{\prime}}$, $(Y, \sigma)$ is a symplectic manifold, where $\sigma$ is the form induced by $d \omega^{\prime}$. Finally, $M$ is identified with $I \times M^{\prime}$ where $M^{\prime}$ is an isotropic submanifold of $Y$ and we may finish the proof by applying theorem 1 in the same way as in the proof of proposition 1 .

4. Maximum modulus manifolds of maximal dimension. From now on, we shall denote by $D$ a strictly pseudoconvex domain with real analytic boundary in $\mathbb{C}^{n}$. If $M$ is a real analytic submanifold of the boundary we shall denote by $M^{c}$ a complexification of $M$.

Proposition 3 [HS]. Let $M$ be a submanifold of $\partial$ D which is $(L P H)$. Then $M$ is complex-tangential. In particular $M$ is totally real and $\operatorname{dim} M \leq n-1$.

Proof. Let $p \in M$ and let $\rho$ be a defining function for $D$ in a neighborhood of $p$. Let $z=\left(z_{1}, \ldots, z_{n}\right), z_{j}=x_{j}+i y_{j}$ be local coordinates in a neighborhood of $p$ such that $p=0$ and $\rho(z)=x_{n}+O\left(|z|^{2}\right)$. Let $f$ be a holomorphic function in a neighborhood $U$ of $p$ such that $f=0$ on $M \cap U$ and $\operatorname{Re} f<0$ on $\bar{D} \cap U \backslash M$. By the Hopf lemma we have $\partial \operatorname{Re} f / \partial x_{n}(0) \neq 0$. Since the origin is a local maximum for $\operatorname{Re} f$ we have

$$
\frac{\partial \operatorname{Re} f}{\partial x_{j}}(0)=\frac{\partial \operatorname{Re} f}{\partial y_{j}}(0)=0, \quad 1 \leq j \leq n-1, \quad \frac{\partial \operatorname{Re} f}{\partial y_{n}}(0)=0
$$

and by the Cauchy-Riemann equations we have also

$$
\frac{\partial \operatorname{Im} f}{\partial x_{j}}(0)=\frac{\partial \operatorname{Im} f}{\partial y_{j}}(0)=0, \quad 1 \leq j \leq n-1, \quad \frac{\partial \operatorname{Im} f}{\partial x_{n}}(0)=0, \quad \frac{\partial \operatorname{Im} f}{\partial y_{n}}(0) \neq 0 .
$$

It follows that $\Sigma=\{z \mid \rho(z)=\operatorname{Im} f(z)=0\}$ is in a neighborhood of the origin a manifold of dimension $2 n-2, T_{0}(\Sigma)=\left\{z \mid z_{n}=0\right\}=T_{0}^{c}(\partial D)$ and since $M \subset \Sigma$, $M$ is complex-tangential.

THEOREM 2 [BS]. Let $M$ be a real analytic totally real submanifold of dimension $n-1$ of $\partial D$. Then $M$ is complex-tangential if and only if there exists $M^{c}$ such that $M^{c} \cap \bar{D}=M$. 
Proof. Let $p \in M$ and let $z=\left(z_{1}, \ldots, z_{n}\right), z_{j}=x_{j}+i y_{j}$ be holomorphic coordinates in a neighborhood of $p$ such that $p=0$ and $M=\left\{z \mid y_{1}=\ldots=\right.$ $\left.y_{n-1}=z_{n}=0\right\}$. Let $\rho$ be a strictly plurisubharmonic defining function for $\mathrm{D}$ in a neighborhood of $p$.

Let us suppose that $M^{c} \cap \bar{D}=M$. We have $\rho\left(z_{1}, \ldots, z_{n-1}, 0\right) \geq 0$ and $\rho\left(x_{1}, \ldots, x_{n-1}, 0\right)=0$. In particular $\frac{\partial \rho}{\partial z_{j}}\left(x_{1}, \ldots, x_{n-1}, 0\right)=0, j=1, \ldots, n-1$ and $M$ is complex-tangential.

Conversely, let $M$ be a complex-tangential submanifold of $\partial D$.

We denote $z^{\prime}=\left(z_{1}, \ldots, z_{n-1}\right), z^{\prime}=x^{\prime}+i y^{\prime}$. Since $M$ is complex-tangential, we have $\frac{\partial \rho}{\partial z_{j}}\left(x^{\prime}, 0\right)=0, j=1, \ldots, n-1$. So

$$
\rho\left(z^{\prime}, 0\right)=\frac{1}{2} \sum_{j, k=1}^{n-1} \frac{\partial^{2} \rho}{\partial y_{j} \partial y_{k}}\left(x^{\prime}, 0\right) y_{j} y_{k}+O\left(|y|^{3}\right) .
$$

Since $\frac{\partial \rho}{\partial z_{j}}\left(x^{\prime}, 0\right)=0$, we have also $\frac{\partial^{2} \rho}{\partial x_{j} \partial x_{k}}\left(x^{\prime}, 0\right)=\frac{\partial^{2} \rho}{\partial x_{j} \partial y_{k}}\left(x^{\prime}, 0\right)=0$, for every $j, k=1, \ldots, n-1$, so $\frac{\partial^{2} \rho}{\partial y_{j} \partial y_{k}}(0)=4 \frac{\partial^{2} \rho}{\partial z_{j} \partial \bar{z}_{k}}(0)$. Since $\rho$ is strictly plurisubharmonic, there exits $m>0$ such that

$$
\sum_{j, k=1}^{n-1} \frac{\partial^{2} \rho}{\partial y_{j} \partial y_{k}}\left(x^{\prime}, 0\right) y_{j} y_{k} \geq m\left|y^{\prime}\right|^{2}
$$

for every $\left(x^{\prime}, y^{\prime}\right)$ in a neighborhood of the origin, so $\rho\left(z^{\prime}, 0\right) \geq 0$ and $\rho\left(z^{\prime}, 0\right)=0$ if and only if $y^{\prime}=0$ for $\left|y^{\prime}\right|$ small enough.

Proposition 4 [I2]. Let $M$ be a submanifold of $\partial D$ which is $(L M H)$. Than $M$ is totally real. If we suppose that $M$ is transverse to $T^{c}(\partial D)$, then $M$ admits a foliation by complex-tangential submanifolds of codimension 1.

Proof. Let $p \in M$ and $f$ a holomorphic function in the neighborhood $U$ of $p$ such that $|f|=1$ on $M \cap U$ and $|f|<1$ on $\bar{D} \cap U \backslash M$. Let $z=\left(z^{\prime}, z_{n}\right), z^{\prime}=$ $\left(z_{1}, \ldots, z_{n-1}\right), z_{j}=x_{j}+i y_{j}$ be holomorphic coordinates in a neighborhood of $p$ such that $p=0$ and $D$ has a strictly plurisubharmonic defining function $\rho=x_{n}+h\left(z^{\prime}, y_{n}\right)$, where $h$ vanishes to second order at the origin.

Let $g=\log f$, with $g$ holomorphic in the neighborhood of $p$ and $\operatorname{Im} g(p)=0$. As in the proof of proposition 3 we have

$$
\begin{gathered}
\frac{\partial \operatorname{Re} g}{\partial x_{j}}(0)=\frac{\partial \operatorname{Re} g}{\partial y_{j}}(0)=\frac{\partial \operatorname{Im} g}{\partial x_{j}}(0)=\frac{\partial \operatorname{Im} g}{\partial y_{j}}(0)=0, \quad 1 \leq j \leq n-1, \\
\frac{\partial \operatorname{Re} g}{\partial y_{n}}(0)=\frac{\partial \operatorname{Im} g}{\partial x_{n}}(0)=0, \quad \frac{\partial \operatorname{Re} g}{\partial x_{n}}(0) \neq 0, \quad \frac{\partial \operatorname{Im} g}{\partial y_{n}}(0) \neq 0 .
\end{gathered}
$$

So we may consider the holomorphic change of coordinates near 0 given by $w_{n}=g$, $w_{n}=u_{n}+i v_{n}$ and we have $h\left(z^{\prime}, v_{n}\right) \geq 0$ and $h\left(z^{\prime}, v_{n}\right)=0$ if $\left(z^{\prime}, v_{n}\right) \in M$. It follows that $\operatorname{gradh}\left(z^{\prime}, v_{n}\right)=0$ if $\left(z^{\prime}, v_{n}\right) \in M$. 
Since $h\left(z^{\prime}, 0\right)$ is strictly plurisubharmonic, by [HW] there exists a complex linear change of the coordinates $z^{\prime}$ such that

$$
h\left(z^{\prime}, 0\right)=\sum_{1}^{n-1}\left(1+\lambda_{j}\right) x_{j}^{2}+\left(1-\lambda_{j}\right) y_{j}^{2}+O\left(\left|z^{\prime}\right|^{3}\right), \quad \text { with } \lambda_{j} \geq 0 .
$$

It follows that $\left\{z \mid \rho=0, \frac{\partial \rho}{\partial x_{j}}(z)=0, j=1, \ldots n-1\right\}$ is a totally real manifold of dimension $n$ in a neighborhood of the origin which contains $M$. Suppose that $M$ is not complex-tangential. Than the set $M_{a}=\{z \in M \mid \operatorname{Im} g=a\}$ is a manifold of codimension 1 in a neighborhood of the origin for every $a$ small enough. But $M_{a}$ is $(L P H)$ for the function $F=e^{-i a}\left(f+e^{i a}\right) / 2[\mathrm{DS}]$ and by proposition 3 it follows that $M$ admits a foliation by complex-tangential submanifolds of codimension 1 .

ExAMPLE 1. Let $D$ be the domain

$$
D=\left\{z=\left(z_{1}, \ldots, z_{n}\right) \in \mathbb{C}^{n} \mid \operatorname{Re} z_{n}+\sum_{i=1}^{n-1}\left(\operatorname{Re} z_{i}\right)^{2}+\left(\left(\operatorname{Im} z_{n}+\left(\operatorname{Im} z_{1}\right)^{2}\right)^{4}<0\right\}\right.
$$

and $M=\left\{z \mid \operatorname{Re} z_{1}=\ldots=\operatorname{Re} z_{n}=0, \operatorname{Im} z_{n}=-\left(\operatorname{Im} z_{1}\right)^{2}\right\} . D$ is a strictly pseudoconvex domain with real analytic boundary and $M$ is a totally real submanifold of dimension $n-1$ of $\partial D$. We have $\operatorname{Re} z_{n} \leq 0$ on $\bar{D}$ and $\operatorname{Re} z_{n}=0$ on $\bar{D}$ if and only if and only if $z \in M$. So $M$ is $(L M H)$ for the function $\exp \left(z_{n}\right)$. But

$$
T_{0}(M)=\left\{z \mid \operatorname{Re} z_{1}=\ldots=\operatorname{Re} z_{n-1}=z_{n}=0\right\} \subset T_{0}^{c}(\partial D)=\left\{z \mid z_{n}=0\right\}
$$

and $M$ is transverse to $T_{z}^{c}(\partial D)$ if $\operatorname{Im} z_{1} \neq 0$.

COROLlary 1 [DS]. Let $M$ be an n-dimensional real analytic submanifold of $\partial D$ which is $(L M H)$. Then $M$ is totally real and admits a real analytic foliation of codimension 1 by complex-tangential submanifolds.

THEOREM 3 [DS]. Let $M$ be an $n$-dimensional real analytic totally real submanifold of $\partial D$. Then $M$ is $(L M H)$ if and only if $M$ admits a real analytic foliation of codimension 1 by complex-tangential submanifolds.

P r o of. By corollary 1 we have only to prove the converse. Let $z \in M$ and suppose that $M_{a}=\{z \in M \mid \varphi(z)=a\}$ give a foliation of $M$ by complex-tangential submanifolds, where $\varphi$ is real analytic in a neighborhood of $z$ in $M$. Since $M$ is totally real there exists a holomorphic extension $\widetilde{\varphi}$ of $\varphi$ in a neighborhood of $z$ in $\mathbb{C}^{n}$. Let $\Sigma=\{z \mid \operatorname{Im} \widetilde{\varphi}(z)=0\}$. Since $d \operatorname{Re} \widetilde{\varphi}(z) \neq 0$, by the Cauchy-Riemann equations, $\Sigma$ is a hypersurface in a neighborhood $U$ of $z$ which contains $M$. Also if $a \in \mathbb{R}, \Sigma_{a}=\{z \in U \mid \widetilde{\varphi}(z)=a\}$ is a complex submanifold of $\Sigma$ which is a complexification of $M_{a}$. By proposition 4 , we have $\Sigma_{a} \cap \bar{D}=M_{a}$, so $\Sigma \cap \bar{D} \cap U=M \cap U$. It follows that $\operatorname{Im} \widetilde{\varphi}$ has constant sign on $D \cap U$, so $M \cap U$ is $(L M H)$ for one of the functions $\exp ( \pm i \widetilde{\varphi}(z))$.

EXAMPLE $2[\mathrm{DS}]$. Let $B_{2}$ be the unit ball in $\mathbb{C}^{2}, \alpha_{1}, \alpha_{2} \in \mathbb{R}$ such that $\alpha_{1}^{2}+\alpha_{2}^{2}=$ 1 and $\gamma=\alpha_{1}^{2} / \alpha_{2}^{2}$ is irrational and $M=\left\{z=\left(z_{1}, z_{2}\right)|| z_{1}\left|=\alpha_{1},\right| z_{2} \mid=\alpha_{2}\right\}$. $M$ is a 
totally real 2-dimensional submanifold of $\partial B_{2}$ which is foliated by the complextangential curves $M_{c}=\left\{z=\left(z_{1}, z_{2}\right) \mid z_{1}=\alpha_{1} e^{-c i \alpha_{2}^{2} t}, z_{2}=\alpha_{2} e^{c i \alpha_{1}^{2} t}\right\}$. Since $M_{c}$ is dense in $M$, it follows that $M$ is not a global maximum modulus set. But $M$ is $(L M H)$ for the function $f\left(z_{1}, z_{2}\right)=z_{1}^{2} z_{2}^{\gamma}$.

\section{Complex-tangential foliations and diagonalizable Levi form}

Lemma 1 [BI]. Let $M$ be a $k$-dimensional submanifold of $\partial D$. Let $p \in M$ such that $M$ is not complex-tangential in a neighborhood of $p$. Then $T(M) \cap T^{c}(\partial D)$ is a distribution of rank $k-1$ in a neighborhood of $p$.

Proof. Since $M$ is not complex-tangential, we have $\operatorname{dim} T_{z}(M) \cap T_{z}^{c}(\partial D)<k$ for $z$ in a neighborhood of $p$ and since both $T_{z}(M)$ and $T_{z}^{c}(\partial D)$ are subspaces of $T_{z}(\partial D)$ we have $\operatorname{dim} T_{z}(M) \cap T_{z}^{c}(\partial D)=k-1$ for $z$ in a neighborhood of $p$.

THEOREM 4 [BI]. Let $M$ be a submanifold of $\partial D$ and $p \in M$ such that $M$ is not complex-tangential in a neighborhood of $p$. The following are equivalent:

a) There exists a neighborhood of $p$ where $M$ admits a foliation by complextangential submanifolds of codimension 1.

b) There exists a neighborhood $U$ of $p$ such that $\mathcal{L}(\xi, \eta) \in \mathbb{R}$ for any sections $\xi, \eta$ over $U$ of the bundle $T(M) \cap T^{c}(\partial D)$.

Proof. By Frobenius theorem a) is valid if and only if the distribution defined by $T(M) \cap T^{c}(\partial D)$ is integrable. With the notations of 1c) this happens if and only if $j^{*}(d \omega)=\varphi \wedge j^{*}(\omega)$. Since $d \omega(\xi, \eta)=-\frac{1}{2} \operatorname{Im} \mathcal{L}(\xi, \eta)$ for any sections $\xi, \eta$ of $\operatorname{ker} \omega$ we obtain the result.

By theorem 2, proposition 2 and theorem 3 we obtain:

COROllary 2 [BI]. Let $M$ be a real analytic totally real submanifold of $\partial D$ which is not complex-tangential at any point. Then $M$ is $(L M H)$ if and only if $\mathcal{L}(\xi, \eta) \in \mathbb{R}$ for every $\xi, \eta$ sections of $T(M) \cap T^{c}(\partial D)$. This is always true if $\operatorname{dim} M \leq 2$.

Corollary 3 [BI]. Let $M$ be a real analytic totally real $n$-dimensional submanifold of $\partial D . M$ is $(L M H)$ if and only if for every $p \in M$ there exists a complex $\mathcal{L}$-orthogonal frame of $T^{c}(\partial D)$ in a neighborhood of $p$ which generates $T(M) \cap T^{c}(\partial D)$ over $\mathbb{R}$.

ExAmple 3 [I1]. Let $D=\left\{z=\left.\left(z_{1}, z_{2}, z_{3}\right) \in \mathbb{C}^{3}\left|2 \operatorname{Re} z_{3}+\right| z_{1}\right|^{2}+\left|z_{2}\right|^{2}+\left|z_{3}\right|^{2}\right.$ $<0\}$ and $M=\left\{z \in \partial D \mid \operatorname{Re} z_{2}=2 \operatorname{Im} z_{1}, \operatorname{Re} z_{1}=\operatorname{Im} z_{2}\right\} . D$ is isomorphic with the unit ball in $\mathbb{C}^{3}$ and $M$ is a real analytic totally real submanifold of dimension 3 of $\partial D$. The vector space $T_{0}(M) \cap T_{0}^{c}(\partial D)$ is generated by

$$
\xi=\operatorname{Re}\left[\left(\frac{\partial}{\partial z_{1}}\right)_{0}-i\left(\frac{\partial}{\partial z_{2}}\right)_{0}\right] \text { and } \eta=\operatorname{Re}\left[2\left(\frac{\partial}{\partial z_{2}}\right)_{0}-i\left(\frac{\partial}{\partial z_{1}}\right)_{0}\right] \text {. }
$$

We see that $\mathcal{L}_{0}(\xi, \eta) \notin \mathbb{R}$, so $M$ does not admit a foliation by complex tangential submanifolds. 
Theorem 4 [BI]. A real analytic submanifold $M$ of $\partial D$ is $(L P H)$ if and only if $M$ is complex-tangential.

Proof. If $\operatorname{dim} M=n-1$, it follows by proposition 2 that $M$ is the leaf of an $n$-dimensional submanifold $M^{\prime}$ of $\partial D$ which admits a foliation by complex-tangential submanifolds. By theorem $3 M^{\prime}$ is $(L M H)$ and by the proof of proposition 4 it follows that $M$ is $(L P H)$. The general case follows by applying proposition 2 .

\section{Real analyticity for smooth maximum modulus manifolds}

THEOREM $5[\mathrm{NR}]$. Let $D$ be a strictly pseudoconvex domain with real analytic boundary and $E$ a $\left(L M A^{2}\right)$. Then, for every $\zeta \in E$, there exist a neighborhood $U$ of $\zeta$ and a $C^{1}$ map $G$ on $U$, holomorphic in $D \cap U$, such that $G(z)=\bar{z}$ on $E$.

Proof. Let us suppose that in a neighborhood $V$ of $z$ we have $D \cap V=$ $\{z \in V \mid \rho(z)<0\}$ where $\rho$ is strictly plurisubharmonic on $V$. Let $\Sigma=\{(z, \xi) \in$ $\left.\mathbb{C}^{n} \times \mathbb{C P}^{n-1} \mid z \in \partial D \cap V, \xi=[\partial \rho(z)]\right\}$ where $\mathbb{C P}^{n-1}$ is the complex projective space of dimension $n-1$, and $[\partial \rho(z)]$ is the point in $\mathbb{C P}^{n-1}$ which has homogeneous coordinates $\left(\partial \rho / \partial z_{1}, \ldots, \partial \rho / \partial z_{n}\right)$. By [WEB] it follows that $\Sigma$ is a real analytic totally real submanifold of dimension $2 n-1$ of $\mathbb{C}^{n} \times \mathbb{C P}^{n-1}$. We denote by $\chi=\left(\chi_{1}, \chi_{2}\right)$ the antiholomorphic reflection across $\Sigma$.

Let $z \in E$ and $f$ a holomorphic function in a neighborhood $U$ of $z, U \subset V$, such that $|f|=1$ on $E \cap U$ and $|f|<1$ on $\bar{D} \cap U \backslash E$. By the Hopf lemma we have $[\partial \rho(z)]=[\partial f(z)]$ for every $z \in E$. We denote $G(z)=\overline{\chi_{1}(z,[\partial f(z)])}$. Since $\chi$ is antiholomorphic and $\chi(\Sigma)=\Sigma$ it follows that $G$ is a $C^{1}$ map on $U$, holomorphic in $D \cap U$, and if $z \in E$, we have

$$
G(z)=\overline{\chi_{1}(z,[\partial f(z)])}=\overline{\chi_{1}(z,[\partial \rho(z)]}=\bar{z} .
$$

Corollary $4[\mathrm{NR}]$. Let $D$ be a strictly pseudoconvex domain with real analytic boundary in $\mathbb{C}^{n}$ and $M$ a $C^{1}$ submanifold of dimension $n$ of $\partial D$ which is $\left(L M A^{2}\right)$. Then $M$ is real analytic.

Proof. Let $p \in E$. By theorem 5 there exist a neighborhood $U$ of $p$ and a $C^{1}$ map $G$ on $U$, holomorphic on $D \cap U$ such that $G(z)=\bar{z}$. Then $G$ is a $C^{1}$ diffeomorphism in a neighborhood of $p$ and the maps $G(z)$ and $F(z)=\overline{G^{-1}(\bar{z})}$ are extensions of the restriction of $\bar{z}$ to $M$. But $F$ and $G$ are holomorphic on opposite wedges with edge $M$, so by the edge of the wedge theorem for $C^{1}$ manifolds [R], it follows that the restriction of $\bar{z}$ to $M$ has a holomorphic extension $\Phi=\left(\Phi_{1}, \ldots, \Phi_{n}\right)$ to some neighborhood of $p$. Then from the $2 n$ equations

$$
\operatorname{Re} \Phi_{j}=\operatorname{Re} z_{j}, \quad \operatorname{Im} \Phi_{j}=-\operatorname{Im} z_{j}, \quad j=1, \ldots, n
$$

we can extract $n$ independent equations which define the $n$ dimensional manifold $M$.

EXAmPLE $4[\mathrm{NR}]$. This example will give a smooth curve transverse to the complex-tangent space in the boundary of the unit ball $B_{2}$ in $\mathbb{C}^{2}$ which is locally a maximum modulus set for $A^{\infty}\left(B_{2}\right)$ and it is not real analytic. 
Let $D$ be the unit disk in $\mathbb{C}, h \in A^{\infty}(D)$, such that $h(z)-(1-z)$ vanishes to infinite order at 1 but $h(z)-(1-z)$ does not vanish identically. If $\varepsilon>0$ is small enough, the set $\Gamma_{\varepsilon}=\left\{\left(z_{1}, z_{2}\right) \in \partial B_{2} \mid \bar{z}_{2}=\varepsilon h\left(z_{1}\right)\right\}$ is a smooth curve in $\partial B_{2}$ which has contact to infinite order at $(1,0)$ with the circle $\left\{\left|z_{1}\right|=1, z_{2}=0\right\}$. So, $\Gamma_{\varepsilon}$ is not real analytic. The curve $\Gamma_{\varepsilon}$ can be parametrized by $\left(z_{1}, \theta\left(z_{1}\right)\right)$ where $z_{1}$ belongs to a smooth simple closed curve $\gamma_{\varepsilon}$ in the $z_{1}$ plane. We consider a smooth extension of $\theta$ to the bounded component $\Omega_{\varepsilon}$ of $\mathbb{C} \backslash \gamma_{\varepsilon}$ that we still denote by $\theta$ and we shall denote $\Omega=\left\{\left(z_{1}, \theta\left(z_{1}\right)\right) \mid z_{1} \in \Omega_{\varepsilon}\right\}$. Let us consider the holomorphic vector field

$$
Z=\frac{-\varepsilon h\left(z_{1}\right)}{1-\varepsilon z_{2} h\left(z_{1}\right)} \frac{\partial}{\partial z_{1}}+\frac{\partial}{\partial z_{2}} .
$$

Since $\bar{z}_{2}=\varepsilon h\left(z_{1}\right)$ and $\bar{z}_{1}=\left(1-z_{2} \varepsilon h\left(z_{1}\right)\right) / z_{1}$ on $\Gamma_{\varepsilon}, Z$ is tangent to $\partial B_{2}$ along $\Gamma_{\varepsilon}$ and it defines a holomorphic foliation of the ball near $(1,0)$. For $\varepsilon$ small enough, $Z$ is close to $\partial / \partial z_{2}$, so we may consider new real variables $\left(x_{1}, y_{1}, x_{2}, y_{2}\right)$ in a neighborhood of $(1,0)$ such that $Z=\partial / \partial x_{2}+i \partial / \partial y_{2}$.

Since for $\varepsilon$ small the change of variables is close to the identity and $Z$ is tangent to $\partial B_{2}$ at the points of $\Gamma_{\varepsilon}$, the points in $\Omega$ may be parametrized by $\left(x_{1}, y_{1}\right)$, so each leaf of the foliation defined by $Z$ has a unique point of intersection with $\Omega$. So there exists a retraction $G$ of the neighborhood of $(1,0)$ in $\bar{B}_{2}$ in a neighborhood of $(1,0)$ in $\bar{\Omega}$ such that the points in $\bar{B}_{2} \backslash \Gamma_{\varepsilon}$ correspond to points in $\Omega$. Using $G$, we can define an almost complex structure $J$ on $\bar{\Omega}$ induced by the complex structure in $\mathbb{C}^{2}$. This structure is integrable because of the complex dimension 1 . So there exists a conformal transformation $f$ from $(\Omega, J)$ to $D$ which extends smoothly to $\bar{D}$. Then in a neighborhood of $(1,0)$, we have $|f \circ G|<1$ on $\bar{B}_{2} \backslash \Gamma_{\varepsilon}$ and $|f \circ G|=1$ on $\Gamma_{\varepsilon}$.

EXAMPLE 5 [NR]. We shall give an example of a smooth curve $\Gamma$ in the boundary of the unit ball $\mathrm{B}_{2}$ in $\mathbb{C}^{2}$ such that the restriction of $\bar{z}$ to $\Gamma$ has a holomorphic extension to $\mathrm{B}_{2}$, but $\Gamma$ is not $\left(L M A^{2}\right)$.

Let $D$ be the unit disk in $\mathbb{C}$ and $h \in A^{\infty}(D)$ such that $h$ vanishes to infinite order at $(1,0)$. Let $\Gamma=\left\{\left(z_{1}, z_{2}\right) \in \partial B_{2} \mid \bar{z}_{2}=2 z_{2}+h\left(z_{1}\right)\right\}$. Since $\Gamma$ has contact to infinite order at $(1,0)$ with $\left\{\left(z_{1}, z_{2}\right)|| z_{1} \mid=1, z_{2}=0\right\}$, it follows that $\Gamma$ is not real analytic. On $\Gamma$ we have

$$
\bar{z}_{1}=\frac{1-2 z_{2}^{2}-z_{2} h\left(z_{1}\right)}{z_{1}}
$$

so the restriction of $\bar{z}$ to $\Gamma$ has a holomorphic extension in a neighborhood of $(1,0)$.

Let us suppose that there exist a neighborhood $U$ of $(1,0)$ and $F \in A^{2}\left(B_{2} \cap U\right)$ such that $|F|=1$ on $\Gamma \cap U$ and $|F|<1$ on $\left(\bar{B}_{2} \backslash \Gamma\right) \cap U$. Using the Hopf lemma as in the proof of proposition 3 , we have $\frac{\partial F}{\partial z_{1}}(1,0) \neq 0, \frac{\partial F}{\partial z_{2}}(1,0)=0$ and $\left(\frac{\partial F}{\partial z_{2}}(z),-\frac{\partial F}{\partial z_{1}}(z)\right) \in T_{z}^{c}\left(\partial B_{2}\right)$ if $z \in \Gamma$. So, if $z \in \Gamma$, we have

$$
\frac{\frac{\partial F}{\partial z_{2}}}{\frac{\partial F}{\partial z_{1}}}=\frac{\bar{z}_{2}}{\bar{z}_{1}}=\frac{z_{1}\left(2 z_{2}+h\left(z_{1}\right)\right)}{1-2 z_{2}^{2}-z_{2} h\left(z_{1}\right)} .
$$


Let $\lambda(t)=\left(\lambda_{1}(t), \lambda_{2}(t)\right)$ be the solution of the Cauchy problem

$$
\frac{d \lambda}{d t}=\left(-\frac{\frac{\partial F}{\partial z_{2}}}{\frac{\partial F}{\partial z_{1}}}(\lambda(t)), 1\right), \quad \lambda(0)=(1,0) .
$$

By the form above of $\frac{\partial F}{\partial z_{2}} / \frac{\partial F}{\partial z_{1}}$, since $\Gamma$ is not real analytic, we have $\lambda_{1}(t)=1-t^{2}+$ $o\left(t^{2}\right)$. Since $\lambda_{2}(t)=t$, we have $|\lambda(t)|<1$ for $t$ small enough, $t \neq 0$, so $\lambda$ is a curve in $\bar{B}_{2}$ through $(1,0)$ and $\lambda(t) \in B_{2}$ if $t \neq 0$. But $\frac{d}{d t}(F \circ \lambda)=0$, so $F$ is constant on $\lambda$. It follows that $|F|=1$ for some points in the ball, which is a contradiction.

\section{References}

[BI] L. Boutet de Monvel et A. Iordan, Sur les feuilletages $\mathbb{C}$-tangents des sousvariétés du bord d'une variété complexe, Astérisque 217 (1993), 39-52.

[BS] D. Burns and E. L. Stout, Extending functions from submanifolds of the boundary, Duke Math. J. 43 (1976), 391-404.

[CC1] J. Chaumat et A. M. Chollet, Ensembles pics pour $A^{\infty}(D)$, Ann. Inst. Fourier 29 (3) (1979), 171-200

[CC2] - - - Caractérisation et propriétés des ensembles localement pics de $A^{\infty}(D)$, Duke Math. J. 47 (1980), 763-787.

[DS] Th. Duchamp and E. L. Stout, Maximum modulus sets, Ann. Inst. Fourier 31 (3) (1981), 37-69.

[FH] J. E. Fornaess and B. S. Henriksen, Characterisation of global peak sets for $A^{\infty}(D)$, Math. Ann. 259 (1982), 125-130.

[HS] M. Hakim et N. Sibony, Ensembles pics dans des domaines strictement pseudoconvexes, Duke Math. J. 45 (1978), 601-617.

[HW] F. R. Harvey and R. O. Wells Jr., Zero-sets of non-negative stricly plurisubharmonic functions, Math. Ann. 201 (1973), 165-170.

[HT] G. M. Henkin and A. E. Tumanov, Interpolation submanifolds of pseudoconvex manifolds, in: Proc. Seventh Winter School, Drogobych 1974, Theory of functions and functional analysis, Central. Ekonom.-Mat. Inst. Akad. Nauk SSSR, Moscow, 74-86 (1976); English transl. in Amer. Math. Soc. Transl. 115 (2) (1980), 59-69.

[11 A. Iordan, A characterisation of totally real generic submanifolds of strictly pseudoconvex boundaries admitting a local foliation by interpolation submanifolds, Math. Ann. 288 (1990), 505-510.

[I2] - Maximum modulus sets in pseudoconvex boundaries, J. Geometric Anal. 2 (4) (1992), 327-349.

[NR] A. Nagel and J. P. Rosay, Maximum modulus sets and reflection sets, Ann. Inst. Fourier 41 (2) (1991), 431-466.

[R] J. P. Rosay, A propos de wedges et d'edges et de prolongements holomorphes, Trans. Amer. Math. Soc. 297 (1986), 63-72.

[WEB] S. Webster, On the reflection principle in several complex variables, Proc. Amer. Math. Soc. 71 (1978), 26-28.

[WEI] A. Weinstein, Lectures on Symplectic Manifolds, CBMS Regional Conf. Ser. in Math. 29, Amer. Math. Soc., Providence, R.I., 1977. 